\title{
Design of Data Sampling Terminal in Telemedicine System
}

\author{
Li Hua-guang ${ }^{1, a,{ }^{*}}$, Fan Yong-hong ${ }^{1, b}$ and An Fei-fei ${ }^{1, c}$ \\ ${ }^{1}$ Qingdao Huanghai College, Qingdao, Shandong, China \\ a252616681@qq.com, ${ }^{\mathrm{b}}$ 164315596@qq.com, ${ }^{\mathrm{c}}$ 369521935@qq.com
}

Keywords: Telemedicine, ECG collecting, Temperature collecting.

\begin{abstract}
In this paper a method of Data Sampling Terminal design is introduced. The system consists of four main parts: temperature collecting, ECG collecting, the main control circuit, and Bluetooth communication module. Another, it also consists of data processing software. Body temperature acquisition system uses NTC type thermostat as a sensor to collect body temperature; ECG collecting uses metal plate electrode as sensors and the collected ECG is amplified and filtered, then it is filtered by digital filters. We will get the high reliability of ECG waveform. The main control circuit includes real-time clock, data storage, LCD display, low-power alarm and communication interface, etc. The main control circuit uses SCM MSP430C1351 as core. The design is also equipped with LCD module, and it can offer customers real-time ECG waveform and temperature. Data stored can store users' physical information, and doctors can make use of them to diagnose diseases. The system can communicate with the monitoring center computer through the serial port and USB port. Bluetooth communication module is used in the communications between Acquisition terminal and GPRS module.
\end{abstract}

\section{Introduction}

With the improvement of people's living standard, the requirement of modern medical treatment is increasing. Many diseases have a large burst, harm, and many of the disease has a strong randomness, which requires a very high efficiency of rescue. At the same time, due to the high population density, large number of population, and the aging of the increasingly serious, how to protect people's proper medical treatment will be an urgent problem to solve. The development of mobile communication technology paved the way for telemedicine, as communication technology development, the development of telemedicine and related technologies will enter a period of rapid development, telemedicine technology will also increasingly mature and perfect. Remote medical system is the use of modern communication technology and sensor technology real-time monitoring of the user's physiological parameters, and real-time to send the collected parameters to the monitoring center. The system shortens the distance between the doctor and the patient, the doctor to provide timely medical services to patients by observing the data returned. The scope of the monitoring system can be monitored not only within the hospital, but also in any place where the communication network can be reached. With the increasing aging of society, remote mobile medical treatment can solve this problem. The system real-time monitoring of the physiological parameters of the elderly and send back the corresponding data, care centers of medical personnel according to the data to determine the health of the elderly, and in an emergency to provide emergency measures. Telemedicine system is developed with the development of the society and the people's demand for health care. The telemedicine system will also be with the development of technology and breakthrough development.

\section{Design of remote medical system}

The telemedicine system is composed of a mobile unit, a GPRS network and a monitoring center. The mobile unit is composed of GPRS module and data acquisition module. The mobile unit block diagram is shown in Fig.1 . 


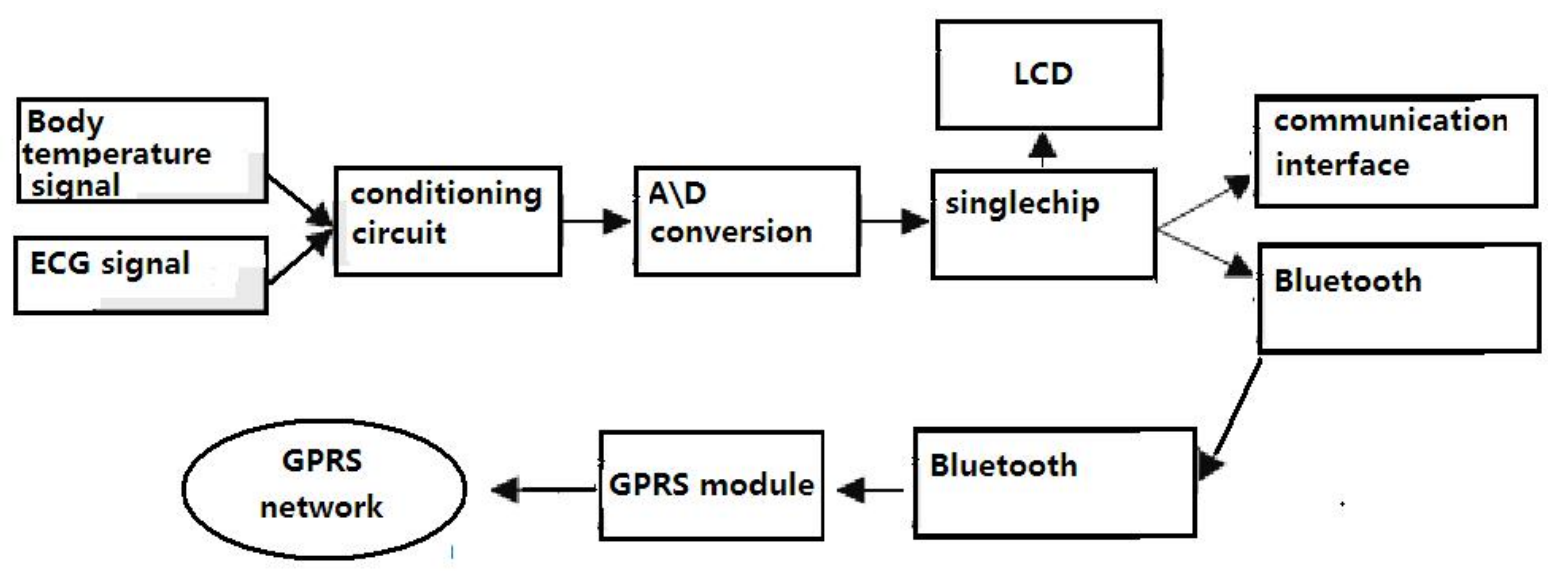

Fig.1 The mobile unit block diagram

\section{Design of temperature acquisition system}

The important index of the patient's condition is the temperature, the accuracy of the temperature measurement is very important to the diagnosis, treatment and nursing of the disease. This system mainly uses the small size, low cost NTC type thermistor as a temperature sensor. The thermistor has advantages of fast response and high sensitivity. Because the temperature characteristic of NTC type thermistor has serious nonlinear reason, it is necessary to carry out high precision linear compensation. In terms of the circuit hardware, the collection side using the serial parallel resistance; in the software, the use of linear interpolation method to compensate. So the acquisition system mainly includes nonlinear compensation (including hardware and software), the temperature signal amplification and the level of the adjustment of the three part, A/D conversion finally.

The thermistor is stable and reliable, and the temperature measurement is fast and sensitive. Therefore, the design of the temperature acquisition part of the sensor, the use of NTC thermistor as a temperature probe. As shown in the Fig.2 is a complete body temperature acquisition circuit.

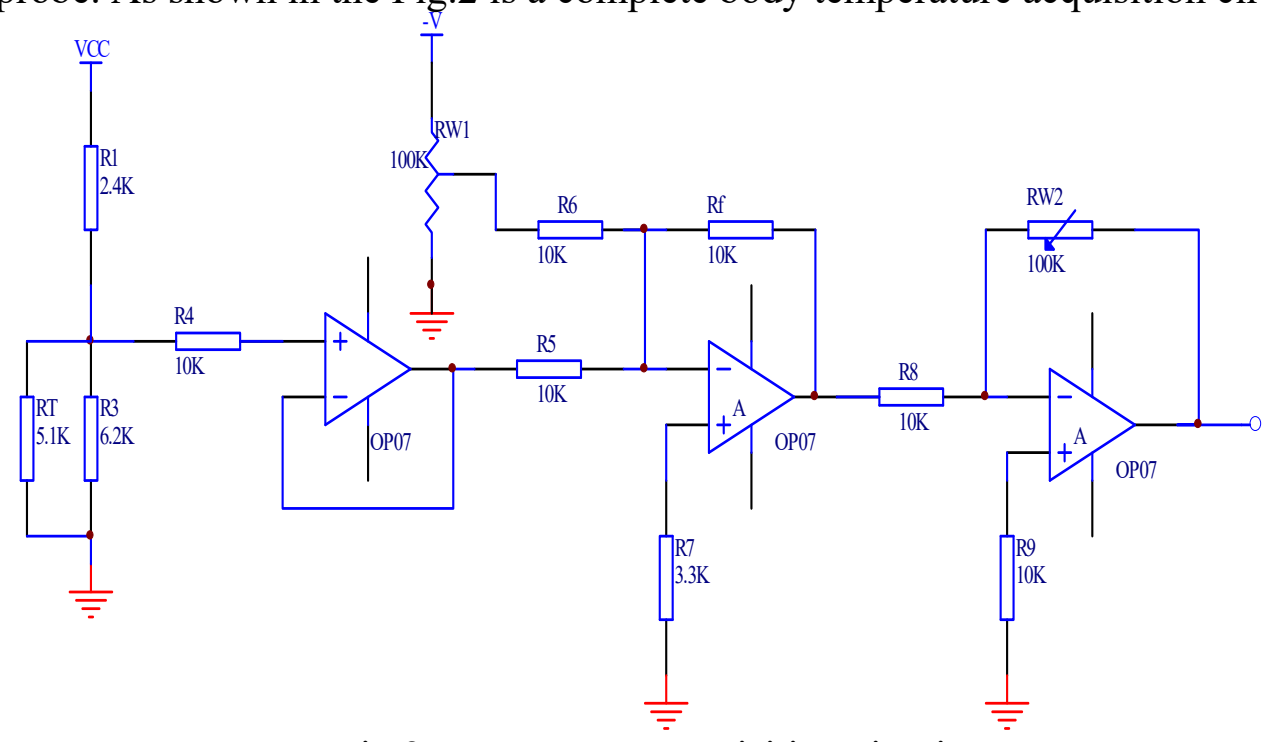

Fig.2 Temperature acquisition circuit

\section{Design of ECG acquisition system}

The current in the circuit can be transformed into the current in the circuit, which can be realized by the biological electrode. The ECG signal collected by the biological electrode is first amplified by the preamplifier, and the weak ECG signal is initially amplified, and the instrument amplifier AD620 is used as a preamplifier. Through the band-pass filter to filter out high frequency and low frequency interference, and then through the main amplifier to further amplify the ECG signal, and then through the $50 \mathrm{~Hz}$ notch circuit to eliminate the power frequency interference, and finally to improve the level and $\mathrm{A} / \mathrm{D}$ conversion. 
The main amplifier circuit is designed to improve the gain for the purpose, the design with the same phase ratio to the operation circuit, as shown in Fig.3.

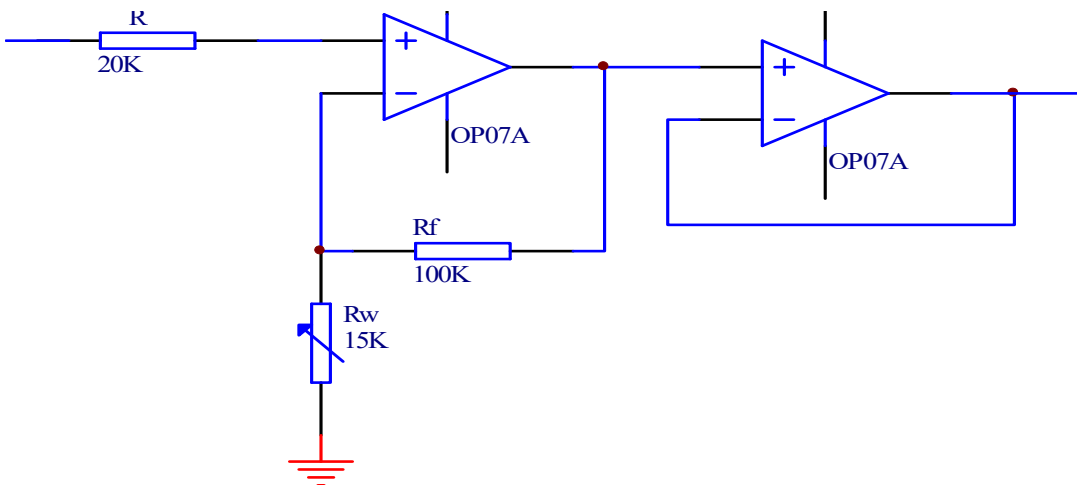

Fig.3 Main amplifier circuit

The same phase scale operation circuit has the advantages of low input resistance and high input resistance. In order to improve operation accuracy, high CMRR of integrated operational amplifier OP07A, common mode rejection ratio as high as $110 \mathrm{~dB}$.

\section{Design of main control system of mobile unit}

The collected signal is sent to the mobile unit master control system, and the main control system of the mobile unit is controlled by a single chip computer. The main control circuit of single chip microcomputer includes: real-time clock, liquid crystal display, single chip microcomputer, data storage and communication interface.

The monolithic integrated circuit is one kind of integrated in the circuit chip, it has the processing ability, the volume is small and so on the merit. This design from the operation ability, power consumption and the actual use of the specific circumstances of consideration, the selection of the MSP430 series microcontroller MSP430C1351.

A/D conversion: To the collecting electrode and after a series of processing the signal is analog signal, vulnerable to interference, in order to improve the signal anti-interference and easy processing of the signal sent, and need to carry out a / D conversion into digital signal. This design selects the frequently used 8 bit analog to digital A/D conversion chip AD0809, the frequency is $250 \mathrm{~Hz}$.

Data storage: In order to allow users to understand the physical condition of any time, you can compress the collected physiological information storage. The stored data allows the monitoring center to understand the situation of the user, to make the correct diagnosis in the event of a sudden. This design with the United States DALLAS research and development of non volatile DS1230 RAM storage capacity, to store the user's physiological information, the capacity of $32 \mathrm{~K}$.

Liquid crystal display circuit: in order to facilitate the user to use, the design with a central wave shape and the current time parameters, liquid crystal display, can display the user's body temperature in real time.

Real time clock circuit: Clock chip by Dallas company production of Juan flow charging clock chip DS1302. It between single chip and can simply adopt synchronous serial communication, needs only three lines: I / O (data line), the rst (reset), SCLK (serial clock). DS1302 work is relatively low power consumption, and keep the clock information and data when the power is less than $1 \mathrm{~mW}$.

Communication interface circuit: this system uses the RS232 interface circuit. RS232 interface is through the COM and MAX232 port communication.

RS232 interface circuit as shown in Fig.4. The circuit is composed of a chip MAX232A and some peripheral circuits, which can convert the TTL/CMOS data from the T1IN and the T2IN input to the RS232 data, and send the data from the T1OUT and the T2OUT to the computer DB9 plug. 


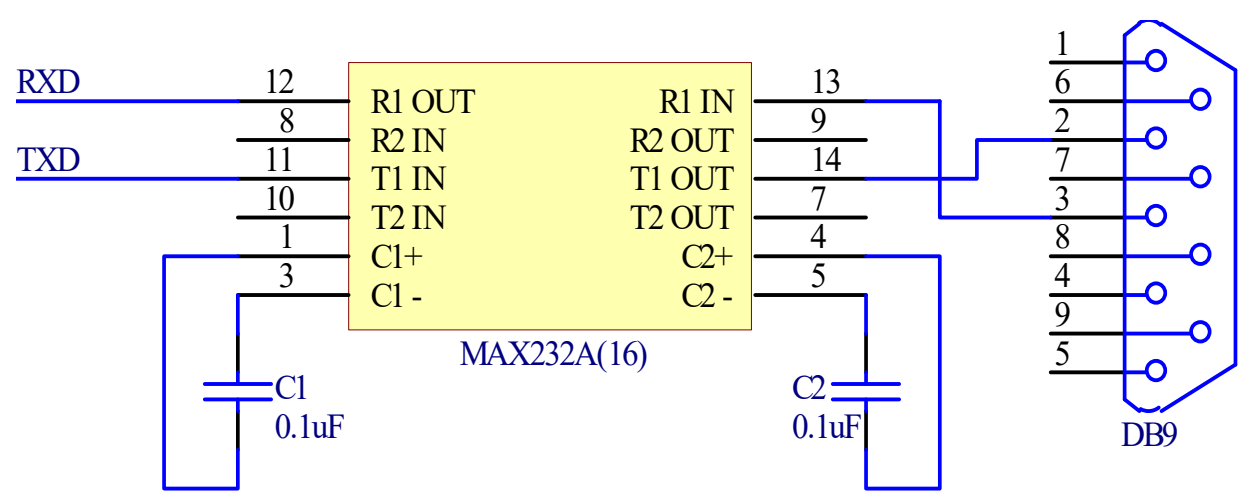

Fig.4 MAX232 interface circuit diagram

The design of Bluetooth module and GPRS module: The communication between GPRS module and a mobile unit here using Bluetooth technology instead of the original fixed connection, the data via Bluetooth to the GPRS module, again through the GPRS module, the collected data are sent to the GPRS network.

\section{Data processing of the data acquisition terminal}

The body temperature signal and the signal transmitted to the amplifying circuit are amplified by the NTC type thermistor and the metal electrode sensor, The amplified analog signal is converted into digital signal by the AD converter circuit, and the signal sent to the LCD screen is sent to the LCD screen. The LCD screen can display the body's current temperature and ECG. When the body temperature or is ECG signal not when in the normal range, the microcontroller starts Bluetooth module and GPRS module, through the GPRS network to the data is transmitted to the hospital receiver, the receiver can display in the abnormal state of body temperature, ECG and body position, fast first aid.

\section{Summary}

In this paper, the design process of data acquisition terminal based on GPRS is introduced. Can the body temperature and ECG signal acquisition, the use of single-chip processing, display to the LCD screen. This design can also be the abnormal signals automatically send exception information to the hospital terminal, medical staff in a timely manner visits, shorten rescue time.

\section{References}

[1] Yin Zhanfang, LV Wenhong, Guo Yinjing. Based on CDMA mobile medical system development. Medical equipment information, in 2002 the three period: 4-7.

[2] Chen Xin, Zhong Shaojun. Mobile medical system GPRS Fujian [J]..2005 seventh computer based on 112-113.

[3] Yin Chong, Hu Junping. A family oriented remote health care system design [J]. The modern electronic technology ", 2008 No. 8: 38-40.

[4] Hua Chengying, Tong Sibai. Fundamentals of analog electronic technology [M]. Tsinghua University, 2006.

[5] Li Jing, Yang Zhou. Portable medical monitor data acquisition terminal design of [J]. electronic design application, 2008. 